\title{
La Investigación Basada en Diseño para propuestas de formación virtual
}

\section{Design-Based Research for virtual training proposals}

\author{
Silvia Coicaud \\ Universidad Nacional de la Patagonia San Juan Bosco - Argentina \\ coicaud.silvia@gmail.com \\ iD 0000-0001-7463-3730
}

Fecha de Recepción: 18 de Junio de 2021

Fecha de Aceptación: 24 de Agosto de 2021

\section{Resumen}

Este artículo pretende generar un análisis teórico acerca de las posibilidades que ofrece la Investigación Basada en Diseño para el campo de la Tecnología Educativa, particularmente para las propuestas de formación virtual. Para ello, se describen los principios y el proceso que implica esta propuesta metodológica y se hace referencia, además, a algunas experiencias concretas que se han desarrollado en diferentes contextos educativos a partir de diseños apropiados que han permitido una real mejora en los proyectos educativos a través de un trabajo colaborativo y comprometido de los actores involucrados. La Investigación Basada en Diseño para propuestas educativas mediadas por tecnologías digitales se focaliza en comprender los procesos de enseñanza y aprendizaje en entornos virtuales, considerando el análisis del contexto como una parte central que no puede ser trivializada. Se investiga diseñando, además, propuestas o soluciones que permiten abordar problemas complejos y situados, analizando su viabilidad como evidencia necesaria para lograr los cambios pretendidos. Esta investigación posibilita hacer contribuciones científicas y prácticas mediante un proceso sistemático e iterativo de diseño, implementación, análisis y rediseño. La filosofía pragmática de esta metodología, en la cual el valor de una teoría radica en su capacidad para producir cambios en el mundo real, constituye una potencialidad tanto para entender y explicar los fenómenos educativos que se suscitan en los entornos tecnológicos actuales, como también para poder desarrollar propuestas de mejora en los procesos y prácticas formativas.

Palabras clave: investigación y diseño, tecnología, educación. 


\section{Abstract}

This article aims to generate a theoretical analysis about the possibilities offered by the Design-Based Research methodology in the field of Educational Technology, particularly for virtual training proposals. For this, the principles and the process involved in this methodological proposal are described, concrete experiences that have been developed in different educational contexts are mentioned, analyzing they are based on appropriate designs that have allowed a real improvement in educational projects through collaborative work and commitment of the members involved. Design-Based Research for educational purposes mediated by digital technologies focuses on understanding teaching and learning processes in virtual environments, considering the analysis of the context as a central part that cannot be trivialized. This investigation is carried out by designing proposals or solutions that allow addressing complex and specific problems, analyzing their viability as necessary evidence to achieve the intended changes. This research makes it possible to make scientific and practical contributions, through a systematic and iterative process of design, implementation, analysis and redesign. The pragmatic philosophy of this methodology, in which the merit of a theory lies in its ability to produce changes in the real world, has a potential both to understand and explain the educational phenomena that arise in current technological environments, as well as to be able to develop proposals for improvement in training processes and practices.

Key words: research and design, technology, education.

\section{Introducción}

Este trabajo tiene como propósito analizar las características que presenta la Investigación Basada en Diseño. Esta metodología se orienta hacia la innovación educativa y pretende, por un lado, indagar sistemáticamente procesos complejos abordando en forma colaborativa diferentes dimensiones y, por otro lado, intervenir en la solución de las problemáticas incorporando nuevos elementos para transformar una situación a partir de una mayor comprensión de esta.

Los actuales programas formativos que utilizan de un modo cada vez más asiduo tecnologías digitales requieren de investigaciones que posibiliten su mejora mediante el trabajo conjunto de todos los actores que participan. Por este motivo, esta metodología adquiere relevancia, pues resulta altamente pertinente en los contextos institucionales que desarrollan propuestas educativas virtuales. 
Desterrando el pensamiento único y asumiendo desafíos en la producción del conocimiento

La ciencia puede concebirse como una empresa humana y cultural que logra ser explicada a partir de comunidades subjetivas de aprendices mutuos que se sostienen recíprocamente. Esto significa que resulta relevante construir discursos válidos desde la ciencia que permitan tanto el relato de la secuencia de acontecimientos que conforman las actividades propias de la producción de conocimientos como también la asunción de principios axiológicos en relación con ellas. No obstante, la producción de conocimientos científicos entraña diversos desafíos. Respecto a las ciencias sociales, la historia de su desarrollo nos revela un camino recorrido en el que han existido distintas posturas teórico-ideológicas, enfoques y tendencias, muchas veces en conflicto.

El pensamiento único con respecto a la investigación científica desconoce sus múltiples propósitos y responsabilidades sociales, lo que condiciona su desarrollo a partir de un modelo emulador y competitivo que genera empobrecimiento cultural y subordinación a parámetros que no resultan apropiados a la idiosincrasia de la producción de conocimientos. Los discursos acerca de la ciencia como herramienta y no como actividad cultural no deberían adoptarse de manera acrítica desde modelos trasplantados.

En el caso de la investigación educativa, las metodologías que se seleccionan pretenden estudiar hechos referentes de la vida de los sujetos de las comunidades educativas: sus comportamientos e historias, los contextos sociales, el funcionamiento de las instituciones, las relaciones e interacciones humanas. Se incorpora en estas investigaciones la voz de las personas participantes y se indaga acerca de sus creencias, actitudes, reflexiones y experiencias a partir de su propia expresión.

El campo de la investigación en las ciencias sociales, en el cual se enmarca la investigación educativa, es transdisciplinar, pues resulta necesaria la intervención de varias disciplinas en la interpretación de los complejos procesos de la vida humana. Además, es una actividad eminentemente política y crítica, que se perfila a través de diferentes posiciones éticas y sociopolíticas. Es importante considerar también que las propuestas de investigación suelen adoptar un enfoque multimétodo en el cual no se privilegia una metodología por encima de otra, sino que se analiza su pertinencia contextual y su relación con el problema que se pretende abordar. Los proyectos de investigación se elaboran como un plan de trabajo flexible, que orienta los procesos de construcción sistemática de conocimientos en relación a los problemas que se intenta estudiar. Se reconstruyen con ello sentidos y significados que son 
puestos en acto por los sujetos en sus prácticas cotidianas. Por ello no existe «un diseño» a priori, sino muchos posibles.

En las propuestas educativas mediadas por tecnologías se requiere de un ir y venir de los datos y las experiencias a la teoría y de esta a aquellos, pues los procesos de investigación no son lineales, sino espiralados, abiertos y recursivos, sin por ello dejar de ser rigurosos y sistemáticos. Desde el momento mismo en que se opta por realizar una investigación, el investigador se inmiscuye en una tarea en la que continuamente deberá elegir y tomar decisiones coherentes y fundamentadas acerca del enseñar y aprender en contextos y ambientes con impronta tecnológica, reflexionando epistemológicamente acerca de estos procesos y comprometiéndose a realizar aportes para conocer y hacer conocer esa realidad educativa, lo que implica la posibilidad de transformarla.

\section{Desarrollo}

La Investigación Basada en Diseño, una propuesta metodológica importante

Se describe a esta metodología como una forma sistemática de investigación, pero al mismo tiempo flexible, que posibilita lograr niveles importantes de comprensión y mejora de los procesos educativos en ambientes complejos a partir de un trabajo iterativo de análisis, diseño, desarrollo e implementación. Este trabajo es realizado de un modo colaborativo por un equipo conformado por investigadores y otros actores. Existen distintas denominaciones y enfoques similares respecto a la Investigación Basada en Diseño, como, por ejemplo, los estudios de diseño, los experimentos de diseño, la investigación colectiva basada en diseño, la investigación de desarrollo, la investigación formativa, las intervenciones programáticas y la investigación de diseño educativo, entre otras (de Benito y Salinas, 2016).

Los diseños que se proponen con esta metodología de investigación precisan sostenerse epistemológicamente, tanto en el conocimiento respecto del campo disciplinario en el que se interviene como en el conocimiento acerca de las teorías pedagógicas explicativas de los procesos esperados. Dado que «los experimentos de diseño son orientados a desarrollar teorías, no meramente a comprobar en forma empírica lo que funciona» (Cobb, Confrey, diSessa, Lehrer y Schauble, 2003, 9), se requiere también conocer el contexto particular para el que se elabora el diseño.

Los aportes que la Investigación Basada en Diseño puede realizar, según Gravemeijer y Cobb (2006), pretenden, por un lado, establecer los resultados 
esperados como casos de fenómenos más generales que puedan ser tenidos en cuenta en otras investigaciones o en otras situaciones de enseñanza. Por otro lado, es necesario ubicar los acontecimientos dentro de contenidos o cuestiones que no hayan sido percibidos, priorizados o tenidos en cuenta con anterioridad, desde los aspectos centrales del diseño realizado. Además, se busca observar y delimitar nuevas categorías científicas, como innovaciones ontológicas que puedan resultar de utilidad para generar o ampliar nuevas alternativas de diseño.

\section{Investigar con diseños en las propuestas de formación virtual}

La formación virtual desarrolla propuestas de enseñanza y aprendizaje desde tecnologías digitales basadas principalmente en sistemas de computadoras y de teléfonos inteligentes a partir de aplicaciones informáticas y de redes telemáticas, implementando para ello construcciones metodológicas alternativas que permitan a docentes y estudiantes interactuar en el ciberespacio sin estar condicionados por limitaciones espaciotemporales. Conocer las problemáticas y posibilidades de mejora de la formación virtual constituye una preocupación relevante para el campo de la Tecnología Educativa, considerando que algunas investigaciones han tenido poca relación con la práctica educativa, fueron muy específicas o de corta duración. Al respecto, Coll $(2008,4)$ plantea, refiriéndose a los estudios acotados de la realidad cuando se incorporan tecnologías de la información y la comunicación:

Los estudios realizados hasta el momento sobre la incorporación de las TIC a la educación escolar y los usos que el profesorado y el alumnado hacen de estas tecnologías muestran con claridad que, en general, las expectativas y los discursos que acabamos de comentar están sensiblemente alejados de lo que ocurre en los centros educativos y en las aulas.

Otra problemática recurrente es que, como afirma Reeves (2011) en algunos trabajos suelen aplicarse métodos de carácter experimental en contextos educativos que son muy complejos, en donde existen variables indefinidas difíciles de aislar. Esto conlleva a la obtención de resultados parciales de investigación, que no generan una comprensión genuina de los procesos educativos que entrejuegan ni tampoco posibilitan una mejora sustancial de estos.

También existen producciones de excelencia en el campo a partir de diseños metodológicos apropiados y de resultados de investigación que propician la discusión disciplinar y el conocimiento acerca de prácticas educativas en contextos diversos. Los métodos etnográficos, las narraciones biográficas, el estudio de casos y la investigación acción, entre otras, son 
algunas de las propuestas metodológicas que posibilitan un abordaje profundo acerca de los procesos educativos en los ambientes actuales de impregnancia tecnológica donde las TIc tienen un rol preponderante. En el caso de la Investigación Basada en Diseño, la creciente producción de trabajos en el campo de la Tecnología Educativa, publicados a partir de esta metodología que comenzó a implementarse fundamentalmente a principios de la década de los noventa, da cuenta de que resulta muy apropiada para investigar propuestas educativas en escenarios virtuales o mediados por tecnologías. Esto se logra a partir de la construcción de productos, de la propuesta de soluciones viables para la mejora y del esclarecimiento acerca del devenir de los procesos y prácticas concretas.

Cabero (2001) plantea que la Tecnología Educativa es una disciplina viva, en constante producción y reformulación, cuya evolución da cuenta de etapas primigenias de fascinación por los medios a otras en donde existe una preocupación por estudiar sistemáticamente los procesos educativos que se generan con la incorporación de las tecnologías digitales. Le compete a la Tecnología Educativa el diseño de medios y de situaciones mediadas de aprendizaje, pero no desde una posición instrumentalista centrada solo en lo técnico o en lo estético, sino desde una perspectiva y dimensión didáctica. Dicha óptica requiere analizar los lenguajes de la mediación como sistemas simbólicos, la pragmática del uso, la utilización crítica y emancipatoria de los productos tecnológicos, la investigación educativa y la evaluación de los procesos, entre otros aspectos (Coicaud, 2016).

En este sentido, la Investigación Basada en Diseño logra generar principios fundamentados para tomar decisiones basadas en la realidad de los procesos en las instituciones educativas que ofrecen formación virtual, que la lleva a delinear, además, políticas educativas factibles. Se supera así la fascinación por los medios tecnológicos y se aborda, de esta manera, una ecología del aprendizaje. Pues los contextos en los cuales este se desarrolla constituyen verdaderos ambientes de interacción, por lo cual el diseño de las intervenciones debería dar cuenta de esta multidimensionalidad situada.

La Investigación Basada en Diseño se focaliza, por lo tanto, en problemas educativos complejos, abordando los contextos reales de las mediaciones tecnológicas. Se busca comprender, por ejemplo, los patrones culturales de un grupo mediante la identificación de creencias y prácticas concretas, los patrones de interacción entre los miembros, los procesos cognitivos que intervienen en la construcción de sistemas semánticos en individuos y grupos, las situaciones en las que se identifican actores, prácticas y actividades, los 
significados de las acciones realizadas, la interacción de docentes y estudiantes con los medios tecnológicos y con el contexto, la articulación entre diferentes tecnologías y su interacción con los criterios didácticos, las estrategias de enseñanza y de evaluación y la relación entre contenidos y actividades con los sistemas simbólicos. Se abordan, entonces, estudios rigurosos y reflexivos para probar y desarrollar, de manera fundamentada, propuestas innovadoras de aprendizaje mediadas por tecnologías, tanto desde la resolución de problemas reales de forma situada como de la construcción y profundización teórica. Es por esto que la fase de difusión de los resultados no se restringe a la elaboración de un informe final de investigación y a su publicación como etapa de culminación del estudio. Por el contrario, los informes se divulgan y se extienden hacia otros ámbitos y actores, lo que genera diferentes miradas a partir de diversas instancias de reflexión crítica y de nuevos focos de interés.

Los principios de diseño que se desarrollan se vinculan de un modo no arbitrario con las posibilidades tecnológicas factibles de ser asumidas por la institución, lo que permite implementar soluciones tangibles a los problemas. Sin embargo, es necesario señalar que esta metodología es reflexiva y opuesta a la racionalidad técnica, pues se asume que los problemas importantes de la formación mediada por las TIC no pueden resolverse con soluciones preconcebidas.

Estos estudios se realizan de forma iterativa. Su carácter recursivo, inacabado y abierto, propio de un proceso en el cual las acciones se revisan y el diseño propuesto se reformula para ajustarlo a las problemáticas analizadas, constituye una prerrogativa para la investigación en un campo complejo y cambiante como lo es la Tecnología Educativa. La influencia que ejercen los diferentes medios tecnológicos sobre el aprendizaje y la enseñanza en ambientes complejos no puede abordarse analizando la tecnología en forma aislada, pues resulta fundamental conocer cómo se integran en los grupos y territorios reales, de qué forma los recursos tecnológicos son interpretados y adaptados y de qué manera inciden los avances tecnológicos en los cambios de las diferentes prácticas sociales. Esto involucra virajes hacia atrás y hacia adelante entre los distintos componentes — propósitos, contexto, marco conceptual, técnicas y preguntas de investigación, diseño, validez- los cuales forman una totalidad integrada e interactiva que genera una estructura metodológica, pero interconectada y flexible. Es importante tener en cuenta que las conexiones entre estos componentes no son taxativas ni conforman reglas que sea preciso seguir a modo de algoritmos, sino que las investigaciones 
basadas en diseño poseen elasticidad y ofrecen posibilidades para su revisión y replanteo.

Se requiere, para ello, de un trabajo colaborativo entre los investigadores y demás actores del proceso educativo - practicantes, docentes, expertos, técnicos, estudiantes, personal de apoyo, directivos- a partir de una participación auténtica que genere implicación a largo plazo. Se respeta y valora el sentido profundo e interno que los sujetos atribuyen a sus acciones y experiencias: sus trabajos, aprendizajes y preocupaciones, el entramado de fuerzas que responden a diversos intereses y el devenir de los hechos desde su propia perspectiva, como una amalgama que posibilita conocer y comprender los procesos educativos. De esta manera, el vínculo entre investigadores y sujetos huye de la lógica de relación entre profesionales expertos y personas comunes, en la que unos son los que saben y los otros son los que ignoran. Se trata, en cambio, de generar comunidades multívocas de diálogo y de construcción conjunta del conocimiento.

\section{El proceso de investigación}

Como planteábamos, la comprensión y mejora de propuestas a través de diseños adecuados es el propósito básico de esta metodología. Este proceso de investigación se realiza en varias etapas que no son lineales, sino recursivas. Matthew, Easterday, Rees Lewis y Gerber (2018) mencionan las siguientes:

- Enfocar: Se lleva a cabo para definir los límites y el alcance del proyecto que se quiere investigar, estableciendo el problema general y la dirección del estudio. Se especifica quiénes serán los implicados en todo el proceso, los roles y las tareas que cumplirá cada uno y los recursos necesarios para asumir el trabajo.

- Entender: Se estudia el contexto, las particularidades de la institución educativa, las demandas y necesidades de los destinatarios, y las propuestas preexistentes. Se pueden emplear para ello diversas técnicas empíricas: la observación, las entrevistas, las encuestas, el análisis de datos; y también recurrir a fuentes secundarias.

- Definir: Aquí se delinea el problema. Se establecen los objetivos, las preguntas de investigación y se analizan las limitaciones. Un problema antes indeterminado adquiere ahora posibilidades de ser abordado.

- Concebir: Es necesario imaginar la solución o la propuesta que permitirá lograr los objetivos. Esto incluye el diseño y análisis de prototipos, sus componentes y relaciones. Esta etapa constituye el corazón de la investigación. 
- Construir: La solución o propuesta se convierte en un prototipo que puede ser utilizado para poner a prueba las ideas concebidas.

- Testear: El diseño se evalúa en el contexto real. También se puede efectuar un testeo paralelo sobre aspectos tales como la relevancia, la consistencia y la implementación práctica.

- Presentar: En este momento se les explica a los destinatarios clave por qué y de qué manera el diseño permite resolver un problema relacionado con sus intereses, mejorar un proyecto educativo o posibilitar una mayor comprensión acerca del mismo para futuras acciones.

Por su parte, De Benito y Salinas (2016) explican, retomando los aportes de Reeves (2000), que más allá del número de etapas que se incluyan en el proceso, todas abordan una serie de acciones comunes. Estos autores mencionan: la definición del problema para analizar la situación y el contexto; el desarrollo de un diseño, fundamentado en un marco teórico; la implementación del diseño, donde se realiza también la recogida de información para su validación; y la evaluación de todo el proceso, con la consecuente producción de documentación y la elaboración de principios de diseño. Durante toda la investigación, se analizan de manera iterativa las etapas que se van desarrollando, lo que posibilita trabajar en el rediseño para lograr una mejora tanto en el cuerpo teórico como en las intervenciones que se realizan.

Rinaudo y Donolo (2010) describen distintas fases concernientes a los aspectos metodológicos en los estudios de diseño: preparación del diseño, implementación y análisis retrospectivo. La primera fase de preparación del diseño consiste en explicitar teóricamente los criterios que justifican las decisiones tomadas. Se definen las metas u objetivos de aprendizaje que se pretende logar con el diseño, se describen las condiciones iniciales y las intenciones teóricas de la investigación, ya sea para convalidar teoría existente o para generar nueva teoría, y se desarrolla un diseño instructivo a partir de una serie de conjeturas que se establecen sobre la forma en que se realizarán los procesos de enseñanza y aprendizaje y los medios que los apoyarán.

La segunda fase es la implementación del experimento de diseño. Aquí, además de poner a prueba un diseño instructivo (microciclo de diseño) para demostrar sus posibilidades, también se intenta validar y mejorar la teoría que fue explicitada en la primera fase, para lograr una mejor comprensión del proceso y efectuar un ajuste continuo del mismo (microciclo de análisis). 
En la tercera fase de análisis retrospectivo, se analizan sistemáticamente los datos recabados, episodio por episodio, considerando como principios de realidad las múltiples variables que intervienen, como el clima y las interacciones entre docentes y alumnos, la cooperación, el compromiso con el aprendizaje, el sistema y la organización institucional, el ambiente del contexto, el apoyo brindado a los alumnos, los contenidos curriculares abordados, el desarrollo profesional, el financiamiento, entre otras. Se reconstruye en esta etapa la teoría pedagógica, lo cual sentará las bases para un nuevo ensayo del diseño. Se revisan también los propósitos iniciales del proyecto de investigación.

Respecto a los métodos para la recolección de los datos, dado que se prevé recabar información acerca de múltiples variables en contextos complejos, se sugiere emplear tanto métodos cualitativos como cuantitativos, con diferentes técnicas e instrumentos. La observación, el análisis documental, los registros narrativos, las entrevistas en profundidad, las encuestas, los cuestionarios, el análisis de contenido, las pruebas estandarizadas, las grabaciones en video (individuales o grupales), el registro de autopercepciones y de intercambios comunicativos son algunos de los procedimientos y técnicas de recolección y análisis que pueden utilizarse.

La calidad en la Investigación Basada en Diseño se establece resguardándola en la teoría a partir de la fundamentación y de las aseveraciones que se esgrimen. Se por metas pedagógicas relevantes para la mejora de una propuesta educativa en un contexto determinado que se indaga; se preocupa por la rigurosidad del diseño desde técnicas de triangulación y un adecuado plan de evaluación situado; se mantiene niveles apropiados de coherencia y racionalidad en la reconstrucción teórica y del diseño, considerando fundamentalmente la perspectiva de los estudiantes (Rinaudo y Donolo, 2010).

Por su parte, Plomp y Nieveen (2009) señalan como criterios de validez, en una fase preliminar, la relevancia del contenido que se selecciona, la consistencia del constructo en el momento del primer diseño, la factibilidad en la sucesión de los prototipos y la eficacia, a partir de la evaluación del impacto desde las iteraciones del proceso. Es importante destacar que, como lo afirman Johnson y Christensen (2017), la combinación de métodos que se emplean en una Investigación Basada en Diseño posibilita una legitimación de validez múltiple. 


\section{Algunas experiencias}

Juan Moreno García (2011) diseñó, desarrolló e implementó un prototipo de entorno virtual para ofrecer recursos de investigación a los alumnos, profesores y tutores de una maestría y un doctorado en Tecnología Educativa de la Universidad de Islas Baleares. Realiza, en primera instancia, una breve reseña contextualizada de las carreras de maestría y doctorado en las que radica su investigación y de las necesidades detectadas, y justifica luego el estudio. En el marco teórico conceptualiza con profundidad varios conceptos, enfatizando el de comunidad virtual. El desarrollo de la investigación le permitió generar un diseño plenamente funcional, ofreciendo una variedad de recursos, herramientas y servicios en un repositorio colaborativo, como así también canales de comunicación y participación que posibilitaron apoyar y favorecer el proceso de formación de investigadores de las carreras de posgrado. Las herramientas diseñadas fueron colaborativas, de información y de comunicación. La Investigación Basada en Diseño se combinó con el modelo ADDIE (análisis, diseño, desarrollo, implementación y evaluación), y con el modelo de gestión de proyectos IPECC (inicio, planificación, ejecución, control y cierre). La combinación de estos modelos se realizó a partir de las siguientes fases, en las que las 2, 3 y 4 no se realizan secuencialmente, sino de modo interactivo, incorporando cambios según las necesidades que surgen:

- Pre Fase 1: Inicio del proyecto (IPECC). Visión general del proyecto, establecimiento de metas, ámbito de aplicación.

- Fase 1: Análisis (ADDIE) y planificación (IPECC). Concreción del alcance del proyecto, organización del plan (calendario y presupuesto) y del equipo de trabajo con tareas específicas y actividades,

- Fase 2: Ejecución del proyecto (IPECC) incluyendo subfases (diseño, desarrollo e implementación del prototipo ADDIE). Se alcanzan las metas, se resuelven problemas y se construye el diseño del proyecto, que se controla (IPECC) para monitorear los cambios y realizar correcciones (calendario, problemas, expectativas y metas)

- Fase 3: Ejecución y control del proyecto (IPECC). Evaluación (ADDIE).

- Fase 4: Cierre del proyecto (IPECC). Entrega, comunicación de resultados, informe y evaluación.

Entre las conclusiones se menciona que se logró desarrollar un entorno funcional con diversos recursos, canales de comunicación y de participación para coadyuvar al proceso de formación de los alumnos investigadores de la maestría y del doctorado, y que respecto al prototipo los usuarios efectuaron una valoración bastante positiva de su utilidad, los recursos y los servicios diseñados para su formación. 
La tesis de Florencia Giletta (2020), Bimodalidad en la formación docente: una propuesta de acompañamiento a las trayectorias de los estudiantes del ISFD Victoria Ocampo de la localidad de Noetinger, presentada en la Universidad Nacional de la Patagonia Austral, se ha elaborado a partir de una Investigación Basada en Diseño, cuyo propósito consistió en reconocer acciones factibles de ser realizadas en el único Instituto Superior de Formación Docente de una localidad de la provincia de Córdoba para disminuir los índices de deserción y abandono mejorando además el rendimiento académico de los estudiantes de los profesorado. Muchos de estos estudiantes tienen dificultades para asistir a clases diariamente, por lo cual se analizó la posibilidad de capacitar y concientizar a docentes y estudiantes sobre el uso de diferentes recursos tecnológicos a través de estrategias metodológicas innovadoras que beneficiaran a todos los integrantes de la institución educativa.

El proceso de investigación se estructuró a partir de las diferentes fases que proponen Rinaudo y Donolo (2010). Durante la preparación del diseño se establecieron sus bases, los objetivos de aprendizaje, se describieron las particularidades del contexto de los alumnos de Noetinger y de la institución educativa. También se especificaron las intenciones teóricas. Luego se elaboró el diseño, con una propuesta de medios y estrategias TIC para llevar a cabo el proceso educativo. Durante la implementación de este se planificó un diseño instructivo en el que se recurrió a distintas técnicas de investigación. En la tercera fase, se realizó un análisis retrospectivo acerca del diseño. Esto permitió efectuar ajustes pertinentes y adecuados a la dinámica y al contexto. Desde esta nueva mirada, se retomaron conceptos relevantes del marco teórico, se reformularon las intencionalidades y algunas actividades que habían sido planificadas inicialmente para la capacitación. Este proceso posibilitó mejorar el diseño formativo basado en la bimodalidad para el ISFD.

La tesis de Victoria Marín Juarros (2014), Modelos de rediseño de acciones formativas en el entorno virtual de enseñanza-aprendizaje. Diseño y experimentación de estrategias metodológicas de integración de los entornos institucionales y abiertos, se realizó a partir de una Investigación Basada en Diseño combinada con el análisis de varios casos de estudio. Se pretendió mejorar pedagógicamente los ambientes virtuales de aprendizaje mediante la integración de entornos personales, sociales e institucionales, indagando acerca de las metodologías más apropiadas para la utilización de entornos abiertos en el contexto formal y sobre la modificación en la concepción del aula en las interacciones didácticas que se generan y en el cambio de roles de docentes y alumnos. 
Se efectuó un análisis de la situación e identificación del problema a partir de investigaciones ya desarrolladas en el uso de TIC en entornos virtuales y de la decisión de mejorar los procesos integrando todos los ámbitos del aprendizaje. Se desarrollaron soluciones desde un marco teórico y una estrategia metodológica basada en el diseño, la investigación-acción y el estudio de casos. Los casos múltiples que se analizaron estaban vinculados con diferentes asignaturas y planes de estudios, incorporando la iteración del primer caso con el quinto. Las etapas que se desarrollaron en la Investigación Basada en Diseño fueron: análisis de la situación e identificación del problema; desarrollo de soluciones: teoría y diseño; implementación de los estudios de casos; validación y evaluación, que se incluyen en la implementación de los estudios de caso; y producción de documentación (informes de los casos) y principios de diseño. Estos principios se elaboraron a partir de las metodologías didácticas para los entornos abiertos de aprendizaje descriptos, de las conclusiones y del informe del cruzado de los casos. Se validó y evaluó este proceso considerando a los actores implicados -alumnos, profesores, observadora participante y no participante - a partir de una taxonomía de evaluación.

\section{Algunas conclusiones}

Los trabajos de investigación realizados en programas de formación virtual que pretenden conocer tanto los desarrollos logrados como las dificultades encontradas por parte de los sujetos intervinientes, con el fin de conocerlos de un modo pormenorizado para proponer mejoras, requieren que el equipo de investigadores efectúe una inmersión en una tarea que demanda aportes colaborativos de los actores implicados, y también decisiones adecuadas al contexto sociopolítico e institucional. En este sentido, la Investigación Basada en Diseño constituye una metodología que resulta apropiada para estudiar los procesos educativos que tienen lugar en las propuestas virtuales de enseñanza y aprendizaje, en donde las mediaciones tecnológicas cumplen un rol fundamental. La posibilidad de indagar las particularidades del contexto y de elaborar un marco teórico de referencia para luego trabajar en un prototipo de diseño que se implementa, se evalúa entre todos los actores que forman parte de la propuesta formativa, se rediseña a partir de los replanteos que el análisis de la realidad establece y de la teoría que se profundiza constituye un modo pertinente de investigar los desarrollos de las propuestas actuales que incorporan tecnologías digitales para la gestión, la enseñanza y el aprendizaje. 
Es importante señalar también que los fenómenos educativos son procesos sociales multidimensionales. Obviamente, gran parte de los problemas en educación no se resuelven con la actividad investigadora. Las prácticas educativas están situadas en contextos sociales y políticos que las condicionan y les otorgan identidad. La creación de conocimientos a partir de la investigación educativa se encuentra con un tren en marcha al que se intenta encauzar, frenar o acelerar, pero sin ser la causa del movimiento. Hay que tener muy en cuenta, por lo tanto, que las alternativas de cambio, innovación y mejora basadas en los resultados de la investigación educativa constituyen propuestas de intervención en procesos que aún están en curso, con historias y tradiciones ya construidas.

Asumimos que no existe un pensamiento único ni tampoco una metodología única y omnipotente para investigar los complejos procesos educativos que se suscitan en la formación virtual. No existe un determinismo en este sentido, porque no hay instrumentos ni métodos infalibles para explicar los hechos de la realidad. El conocimiento surge desde una interacción dialéctica entre el objeto de estudio y la participación de diversas personas, factores e incluso valores y creencias. La Investigación Basada en Diseño es una alternativa válida, fundamentalmente para las propuestas educativas mediadas por tecnologías que requieren ser analizadas y mejoradas por el conjunto de los actores implicados en su gestión y desarrollo, quienes pretenden además generar principios de acción orientadores de futuras decisiones importantes tanto para los proyectos como para las instituciones que los propician.

Conocer el contexto real y la idiosincrasia de estos proyectos, construir y reconstruir un diseño de formación comprendiendo cabalmente sus porqué y para qué a partir del diálogo fecundo y sostenido en la comunidad virtual de práctica constituye una opción desafiante para quienes han asumido un compromiso con el estudio sistemático de los fenómenos educativos, interviniendo además en aumentar su calidad. La posibilidad de combinar el proceso tanto con otras metodologías y estrategias de investigación -por ejemplo, el estudio de casos, los métodos biográficos o el análisis de los datos que nos ofrecen los medios informáticos - como también con otros modelos para el análisis, desarrollo y evaluación de los proyectos es otra de las grandes ventajas que nos presenta la Investigación Basada en Diseño, que resulta flexible y altamente adecuada para las propuestas de formación virtual que existen en la actualidad, las que cada vez adquieren mayor relevancia en nuestras instituciones educativas. 
El enfoque pragmático de esta metodología, en donde el valor de una teoría radica en su capacidad para producir cambios en el mundo real, constituye una potencialidad tanto para entender y explicar los fenómenos educativos que se suscitan en los entornos tecnológicos actuales, como también para poder desarrollar propuestas de mejora en los procesos y prácticas formativas.

\section{Referencias bibliográficas}

Anderson, T. (2005). Design-based research and its application to a call center innovation in distance education. Canadian Journal of Learning and Technology, 31(2), 69-84. http://www.jofde.ca/index.php/jde/article/view/18/552

Easterday, M., Rees Lewis, D. y Gerber, E. (2018). The logic of design research. Learning: Research and Practice, 4(2), 131-160. http://liderazgoescolar.uc.cl/index.php?option=com_content\&view=article\&id= 1057: investigacion-basada-en-el-diseno-en-educacion\&catid=13\&ltemid=291

Escudero-Nahón, A. (2018). Principios de Investigación Basada en Diseño para la creación de un modelo de educación virtual. En Afrontar los retos de la educación en el siglo XXI. pp. 217-232. Horson Ediciones Escolares S. A.

Blessing, L. y Chakrabarti, A. (2009). DRM: A Design Research Methodology. Londres: Springer-Verlag London.

Cabero, J. (2001). Tecnología educativa. Diseño y utilización de medios en la enseñanza. Barcelona: Paidós.

Cobb, P., Confrey, J., diSessa, A., Lehrer, R. y Schauble, L. (2003). Design Experiments in Educational Research. Educational Researcher, 32 (1), pp. 9-13.

Coll, C. (2008). Aprender y enseñar con las TIC: expectativas, realidad y potencialidades. Boletín de la Institución Libre de Enseñanza, 72, pp. 17-40.

De Benito, B. y Salinas, J.M. (2016). La Investigación Basada en Diseño en Tecnología educativa. Revista Interuniversitaria de Investigación en Tecnología educativa, 0, pp. 44-59.

Gravemeijer, K. y Cobb, P. (2006). Design research from a learning design perspective. Educational design research.

Johnson, B. y Christensen, L. (2017). Educational research: Quantitative, qualitative, and mixed approaches. Los Angeles: SAGE.

Marín Juarros, V. (2014). Modelo de rediseño de acciones formativas en el entorno virtual de enseñanza-aprendizaje. Diseño y experimentación de estrategias metodológicas de integración de los entornos institucionales y abiertos. 
Moreno García, J. (2011). Diseño, desarrollo e implementación de un prototipo de entorno virtual para una comunidad de investigadores en formación, [proyecto fin de maestría, Universidad de Islas Baleares].

Plomp, T. y Nieveen, N. (2009). (Eds). An introduction to educational design research: Proceedings of the seminar conducted at the East China Normal University. Shanghai. Enschede: Stichting Leerplan Ontwikkeling.

Reeves, T. (2011). Can Educational Research Be Both Rigorous and Relevant? Journal of the International Society for Design and Development in Education, 4.

Rinaudo, M.C y Donolo, D. (2010). Estudios de diseño. Una perspectiva prometedora en la investigación educativa. RED. Revista de Educación a Distancia, 22. 